\title{
CAMBIO CLIMÁTICO Y SALUD
}

\section{CLIMATE CHANGE AND HEALTH}

Rubén Figueroa ${ }^{1, a}$

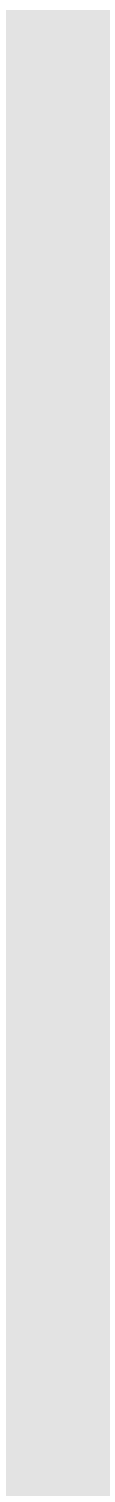

La decisión de la dirección de la Revista Peruana de Medicina Experimental y Salud Pública -RPMESPde privilegiar en el presente número artículos referidos al cambio climático y salud, marca una vez más el importante rol que en la salud pública desempeña este único medio de difusión, ya que el perfil de salud de la población en los próximos decenios estará impactado también por el cambio en el clima del planeta, y del Perú en particular. En este sentido, es de particular trascendencia estratégica difundir lo que los profesionales en el Perú están estudiando e investigando, pues sus conclusiones y recomendaciones constituyen la base de evidencias que deben sustentar las decisiones de política pública en salud.

Lo que se reporta en los artículos aquí publicados son los progresos registrados en el Perú, como una experiencia de importancia para la salud pública, porque se comunican resultados y conclusiones de estudios, posiciones, opiniones y recomendaciones de los autores, para incorporar en la política pública de salud las acciones que deben tomarse para mitigar los efectos negativos del cambio climático en la salud de la población, así como las medidas de adaptación que deben desarrollarse en los próximos años para que los servicios de salud estén mejor preparados con el fin de enfrentar nuevos patrones epidemiológicos en la presentación de las enfermedades que pueden ser impactadas por el cambio climático. Estos estudios abordan el cambio climático y salud desde diferentes perspectivas, todas de igual importancia, y en este espacio, el editor invitado hace mención a algunas de ellas a manera de destacar lo que, a su juicio, expresan uno o varios mensajes que cree son claves para el derrotero que el Ministerio de Salud recorre en la actualidad en su misión ineludible de proponer al país un lineamiento de política de salud que considere los efectos del cambio climático en el perfil de salud de la población y la consecuente capacidad de respuesta del sector para enfrentar esos efectos.

El primer artículo que paso a comentar es el del investigador Carlos A. Sánchez-Zavaleta de la Universidad Peruana de Ciencias Aplicadas, Lima, Perú, sobre la "Evolución del concepto de cambio climático y su impacto en la salud pública del Perú", donde aborda históricamente algunos conceptos y posiciones, sobre todo de instituciones internacionales, acerca del cambio en el clima, y de las vulnerabilidades existentes en la población y sus estructuras de respuesta. Es clara la propia posición del autor en cuanto a que la salida para el sector Salud es la adaptación a los cambios del clima y que la “...mitigación no es una opción costo-efectiva...", así como su propuesta de lo que hay que hacer en salud, independientemente de cual sea el impacto del cambio climático en la salud de la población. Es de alto valor político, más allá de que, precisamente por esa condición de opinión política, requiera de debate ampliado, su conclusión acerca de que el sistema nacional de salud necesita para adaptarse al cambio climático tener como eje la lucha contra la pobreza y la desigualdad mediante la "mejor" identificación de susceptibles, vulnerables o desplazados, "mejorías" en la infraestructura sanitaria, más capacidades en predicción en salud y "mejor" comunicación del riesgo (1).

\footnotetext{
Ministerio de Salud. Lima, Perú

a Editor invitado

Recibido: 16-03-16 Aprobado: 18-03-16
}

Citar como: Figueroa R. Cambio climático y salud [editorial]. Rev Peru Med Exp Salud Publica. 2016;33(1):7-9. doi: 10.17843/rpmesp.2016.331.2006 
Otro de los artículos analizados para este editorial es el de la investigadora Tania Burstein Roda, sobre el "Rol del sector Salud ante el cambio climático", donde reconoce que el cambio climático influye en los determinantes sociales, económicos y ambientales de la salud, entre ellos el aire limpio, el agua segura, y los alimentos suficientes; es clara su posición en cuanto a que el sector Salud no podrá mejorarlos, ni mucho menos removerlos, porque no es su competencia, pero si tiene un rol central para señalar que sin esas mejoras en las condiciones y calidad de vida, la salud de la población se verá muy afectada. En este sentido, considera que el sector Salud debe liderar con el ejemplo la necesidad de reducir la huella de carbono como medida de mitigación en sus instituciones, aunque también señala la ineludible acción que se debe tomar en cuanto a las capacidades de adaptación. Es clave su contribución en cuanto al rol del sector Salud en la rectoría de investigar para superar la pobreza, de información y evidencia que sobre cambio climático y salud existe en el Perú; no hacer generalizaciones sino investigar localmente, enfoques interdisciplinarios y transectoriales, investigar no solo patologías, sino también los factores que las determinan; son los principios rectores que propone al sugerir seis líneas de investigación que deberían desarrollarse ${ }^{(2)}$.

El tercer artículo, "Perú, cambio climático y enfermedades no transmisibles: ¿dónde estamos y a dónde vamos?", lo suscriben el estudiante de Medicina José L. Avilez, la licenciada en Enfermería Janina Bazalar, el cirujano dentista Diego Azañedo y el médico J. Jaime Miranda, de la Universidad Peruana Cayetano Heredia, Lima, Perú y Universidad Católica los Ángeles, Chimbote, Perú. Singular asociación la que realizan los autores entre dos temas asumidos por organismos internacionales como las "mayores amenazas para la salud del siglo XXI": cambio climático por un lado y enfermedades no transmisibles (ENT) por el otro, llamando la atención sobre la insuficiente atención que se les da desde la investigación y desde las políticas públicas. Con excelente raciocinio, lógica y evidencia, los autores relacionan ambas "amenazas" con la urbanización, como ejemplo: actividad física limitada, dietas inadecuadas, aumento del transporte vehicular, son, entre otros, factores que por la contaminación ambiental por gases de efecto invernadero, el sedentarismo y la obesidad, se asocian con la morbilidad por enfermedades cardiovasculares y respiratorias. Pasando por los compromisos derivados del "Llamado de Lima para la acción climática" (COP-20 2014) y el "Acuerdo de París" (COP-21 2015), la formulación por parte del Ministerio del Ambiente del Plan Nacional de Adaptación y las Contribuciones Previstas y Determinadas a Nivel Nacional, donde Salud es uno de los sectores priorizados, se analiza y señala de manera inequívoca como el Perú puede obtener "cobeneficios" para la prevención y control de las ENT a partir de las acciones de mitigación y adaptación que ejecutan otros sectores de gobierno. Concluyen el artículo con buenas recomendaciones para la Academia Nacional de Medicina y para el gobierno, en materia de salud pública ${ }^{(3)}$.

Otro abordaje interesante que da una perspectiva integral a los artículos publicados en el presente número de la RPMESP es el estudio "Potencial vulnerabilidad frente a inundaciones, de los establecimientos de salud públicos de cuatro regiones del norte del Perú", de autoría de Akram Hernández-Vásquez, Hugo Arroyo-Hernández, Guido Bendezú-Quispe, Deys Díaz-Seijas, Stalin Vilcarromero, Juan Rubilar-González, y Edith Gutierrez-Lagos; de la Universidad de Buenos Aires, Argentina; el Instituto Nacional de Salud; la Universidad Nacional San Luis Gonzaga, Ica, Perú; del Instituto Nacional Cardiovascular, EsSalud, Lima, Perú; la Universidad Nacional de la Amazonía Peruana, Iquitos, Perú; la Universidad Mayor, Temuco, Chile y del Ministerio de Energía, Santiago de Chile, Chile. Ante los posibles efectos del fenómeno El Niño (ENSO), los autores consideraron en los departamentos de Tumbes, Piura, Lambayeque y La Libertad los posibles riesgos por inundaciones o deslizamientos de los establecimientos de salud (EE. SS.) del Ministerio de Salud; el análisis espacial se realizó a partir de establecer puntos georreferenciados en un área de influencia concéntrica en un radio de 200, 1000 y 1500 metros. De los 872 establecimientos estudiados, 38 serían afectados por un ENSO con lluvias extremas, siendo Tumbes la región más afectada, puesto que el $37,2 \%$ de sus establecimientos están cerca de los puntos de riesgo; asimismo, entre 2 y $3 \%$ de EE. SS. de las otras tres regiones serían afectados. Alrededor del 30\% de los establecimientos perjudicados corresponden a la categoría I-2 y I-3, es decir, servicios del primer nivel de atención. La simulación realizada permite concluir que considerando a zonas afectadas anteriormente (el estudio tomó datos del ENSO 1997-1998) y estableciendo zonas de riesgo y ubicación geográfica de los EE. SS., se puede identificar aquellos cercanos a puntos de riesgo por inundaciones, con el consiguiente beneficio de prevención si se toman medidas de mitigación. El estudio señala como una limitante importante para acercar la simulación a la realidad, la no disponibilidad de una base de datos actualizada de los EE. SS., así como su falta de georreferenciación ${ }^{(4)}$.

Concluyo esta nota editorial destacando que aun en el escenario adverso del cambio climático, es posible proteger la salud de las personas si hay ejercicio de la responsabilidad política que le cabe al Ministerio de Salud del Perú como organismo rector. En ese sentido, los artículos aquí comentados sugieren pautas claras de política pública que, en su mayoría, son recogidos por la 
propuesta de "Plan integral de mitigación y adaptación frente a los efectos del cambio climático en la salud pública", que fuera encargado a una comisión de trabajo ${ }^{(5)}$ que ya concluyó su elaboración y, por tanto, su aprobación formal por Resolución Ministerial se encuentra en proceso. El Plan tiene el objetivo de establecer las intervenciones estratégicas integrales para la mitigación y adaptación a los efectos del cambio climático en la salud de la población, aunque con una fuerte orientación en la adaptación y con cinco objetivos estratégicos relacionados con lineamientos de política sectorial, generación de evidencia, escenarios de riesgo, participación comunitaria y cuidado integral de la salud. El Ministerio de Salud tiene un gran desafío para la implementación de la propuesta en cuanto a sus principios para darle institucionalidad a la acción necesaria en materia de cambio climático y salud en el Perú.

\section{REFERENCIAS BIBLIOGRÁFICAS}

1. Sánchez Zavaleta CA. Evolución del concepto de cambio climático y su impacto en la salud pública del Perú. Rev Peru Med Exp Salud Publica. 2016; 33(1):128-38.

2. Burstein-Roda T. Rol del sector salud ante el cambio climático. Rev Peru Med Exp Salud Publica. 2016; 33(1):139-42.
3. Avilez J, Bazalar J, Azañedo D, Miranda JJ. P. Perú, cambio climático y enfermedades no transmisibles: ¿Dónde estamos y a dónde vamos?. Rev Peru Med Exp Salud Publica. 2016; 33(1):143-8.

4. Hernández-Vásquez A, ArroyoHernández $\mathrm{H}$, Bendezú-Quispe G, Díaz-Seijas D, Vilcarromero S,
Rubilar-González J, Gutierrez-Lagos E. Potencial vulnerabilidad frente a inundaciones, de los establecimientos de salud públicos de cuatro regiones del norte del Perú. Rev Peru Med Exp Salud Publica. 2016; 33(1):92-9.

5. Resolución Ministerial 246-2014/ MINSA

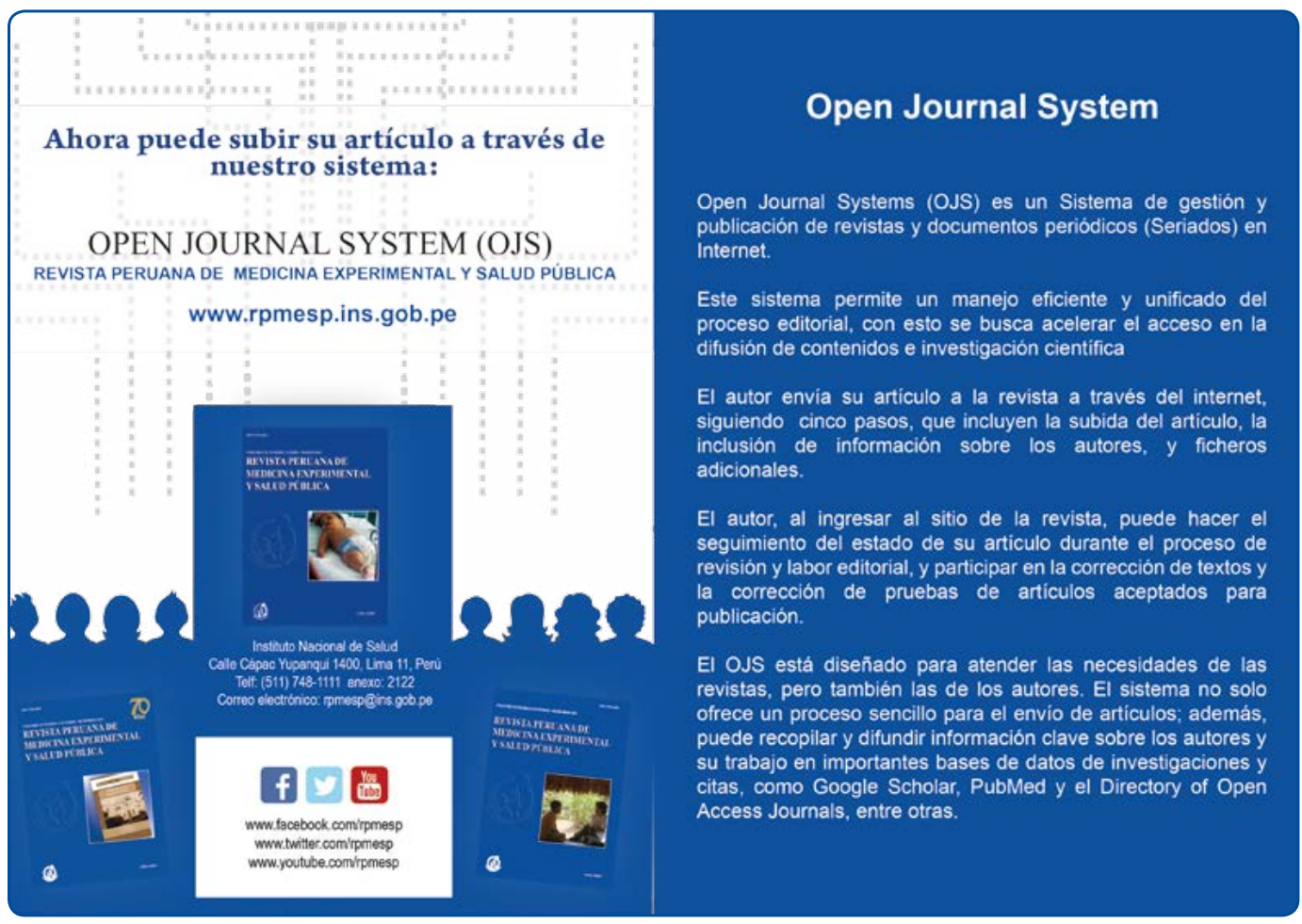

